\title{
Preface to the special collection in honour of Vincenzo Barone
}

\author{
Nino Russo $\cdot$ Nadia Rega $\cdot$ Carlo Adamo
}

Published online: 5 June 2012

(C) Springer-Verlag 2012

\section{The 60th birthday of Vincenzo Barone}

Vincenzo Barone is Full Professor in Theoretical and Computational Chemistry at the Scuola Normale Superiore of Pisa and President of the Italian Chemical Society (SCI).

Vincenzo Barone was born in Ancona (Italy) on November 1952. After his high school education, Enzo (as his friends call him) studied at the Department of Chemistry of the University of Naples, and in 1976, he received his degree (Laurea) in Chemistry discussing the thesis under the supervision of Professor Giuseppe Del Re, one of the pioneers of theoretical chemistry in Italy. He then moved as post-doctoral fellow to the University of Grenoble (France) where he worked under the supervision of

Published as part of the special collection of articles celebrating the 60th birthday of Professor Vincenzo Barone.

\section{N. Russo}

Dipartimento di Chimica, Università della Calabria,

via Pietro Bucci, 87036 Arcavacata di Rende, Italy

e-mail: nrusso@unical.it

\section{N. Rega}

Dipartimento di chimica, Università Degli Studi di Napoli

Federico II, Complesso Universitario Monte S. Angelo

via Cintia, 80126 Naples, Italy

e-mail: nadia.rega@unina.it

\section{Adamo ( $\square)$}

Laboratoire d'Electrochimie, Chimie des Interfaces

et Modélisation pour l'Energie, Ecole Nationale

Supérieure de Chimie de Paris, 11,

rue Pierre et Marie Curie, 75231 Paris, France

e-mail: carlo-adamo@chimie-paristech.fr

\section{Adamo}

Institute Universitaire de France,

103, Boulevard Saint Michel, 75005 Paris, France

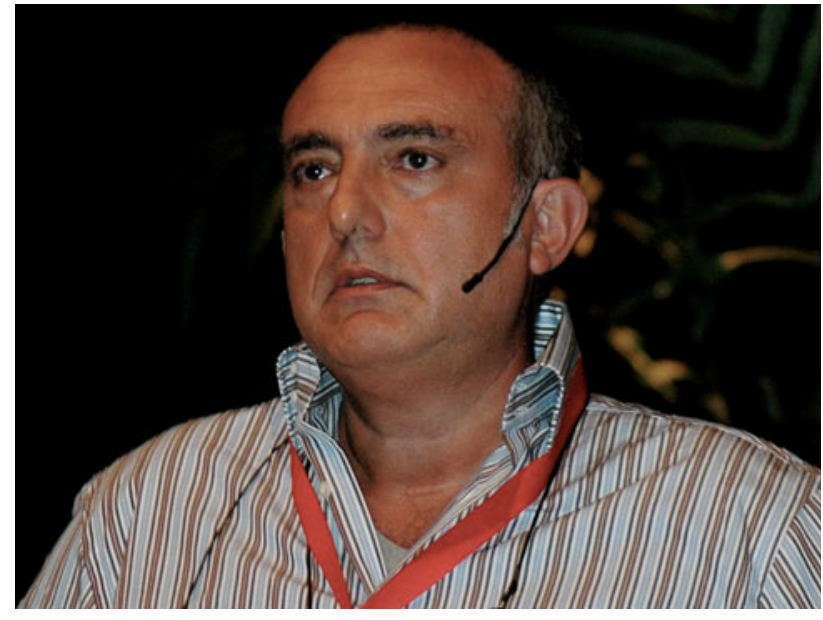

Professor Robert Subra. We can date back to this period his interest in the chemistry of organic radicals, a field in which over the years he has produced major and widely recognized contributions.

From 1978 to 1981, Enzo was Assistant Professor in General Chemistry at University of Calabria (Italy), where he contributed to the creation of the Laboratory of Theoretical and Computational Chemistry (currently led by Professor Nino Russo). During this time, he was visiting scientist in well-known international Laboratories in France (Ecole Normale Supérieure, Paris; Université Joseph Fourier, Grenoble), Canada (Université de Montreal) and Germany (University of Erlangen-Nürnberg).

After a period as Associated Professor at the University of Naples, he become Full Professor of Theoretical Chemistry in 1994. In Naples, Enzo created the Theoretical and Computational chemistry group in which many young researchers found a fertile substrate for learning and working in exciting research fields. In few years, the group reached a remarkable reputation in the international 
scientific community. After this stimulating experience, he moved to Pisa in 2008, firstly as Director of the Institute for chemical and physical processes (IPCF, a Research Institute of the Italian National Research Council) and later as Full Professor in Theoretical and Computational Chemistry at Scuola Normale Superiore, one of the most prestigious universities in Italy.

Also in these two institutions, he attracted countless guest scientists, graduates, post-graduates and post-docs from all over the world. To all the co-workers, Enzo always ensured a congenial atmosphere, allowing the team to work with enthusiasm and efficacy. Many of his former students and post-doc fellows are now professors and/or leading scientists in universities and research centres in Italy and around the world.

In the last years, Enzo has promoted a number of scientific initiatives (e.g. the $\mathrm{M}^{3}$-Village Networks, the Idea Virtual Laboratory and the CODECS COST Action) that have strongly contributed to the development of theoretical and computational chemistry in Italy and in Europe. Furthermore, he has played a seminal role in the creation of the Computational Chemistry Division of the Italian Chemical Society.

His scientific profile is really impressive: he is author of more than 500 papers, which have received over 25,000 citations, with an h-index of 64; in 2009, he was included among the ISI highly cited researchers for the Chemistry category.

Enzo was also invited to hundreds of lectures in national and foreign institutions or congresses, and he is member of the editorial board of prestigious journals (e.g. Phys. Chem. Chem. Phys., Theor. Chem. Acc., J. Comput. Chem.) of the scientific council of many scientific institutions, fellow of the International Academy of Quantum Molecular Science and of the Royal Society of Chemistry, and chairman of different national and international scientific events. Furthermore, he is a co-author of Gaussian, one of the most popular quantum chemistry codes.

Recently, he has received the prestigious Sacconi Medal of the Italian Chemical Society for his contribution to the development of theoretical and computational chemistry.

Of equal importance is the educational activity of Enzo: until now, he has been supervisor of about 20 master and more than $15 \mathrm{PhD}$ students and has directed over 20 postdocs in modern theoretical chemistry research.

Enzo has developed his research activities in many fields of theoretical and computational chemistry, with the overall goal to establish a theoretical microscopic approach for the study of structural, dynamic, spectroscopic and reactive properties of materials, biomolecules and more in general soft matter in complex environments. His contributions to the accurate description of solvent effects and nuclear motions represent a logical element of this strategy.
Of particular interest is Enzo's conception of theoretical and computational chemistry as an "experimental" tool that can be used not only to achieve chemical accuracy in computed physico-chemical properties, but also to unravel and understand phenomena at the atomistic level. This has led Enzo to adopt in his research a strongly multidisciplinary approach, which is focused in the first place on theoretical developments and on multiscale descriptions, but also addresses the potentialities of computer architectures and of grid implementations.

More in details, the areas in which Enzo has provided remarkable contributions include the following:

- Chemical bond and molecular reactivity. In this context, we only mention the work on the absorption of atoms and molecules on solid surfaces, the chemical properties and reactivity of radical species, conformational analysis and photo-electron spectroscopy of biphenyl-like systems;

- Density functional theory (DFT). Already in 1992, Enzo was convinced of the potential of density functional theory to deal with chemical problems. His contributions include the development and validation of new exchange-correlation functionals (such as mPW1PW and PBE0), the validation of DFT for open-shell systems and, more recently, the implementation and parameterization of tight-binding DFT and of its time-dependent extension;

- Solvation theory with special emphasis on the polarizable continuum model (PCM). The approach has been developed and implemented for the evaluation of solvation free energies, including solvent effects on molecular structures in both ground and excited electronic states. In the last years, he has also been involved in the development of a mixed discrete-continuum model especially useful for computational spectroscopy applications. Enzo's work in this field has contributed to make of PCM the most widely used tool to study molecular systems in condensed phases at quantum mechanical level;

- Computational spectroscopy. Enzo's studies on harmonic and anharmonic treatments of molecular vibrations paved the route to unprecedented results in the field. In the last years, his scientific interests have focused on theoretical developments instrumental to the accurate simulation of spectral line-shapes for large molecular systems in condensed phases; as an example of the potential of the approach, one can cite the computation of entire EPR spectra of different molecular systems, in excellent agreement with the experimental counterparts.

This short extract of the Enzo's scientific contributions is inevitably reductive; the interested reader will easily find 
in the current literature his fundamental contributions to the advancement of Theoretical and Computational Chemistry. We are therefore confident that in the next decades, Enzo Barone will continue to develop and apply new theoretical approaches and will thus keep contributing to a better knowledge of Chemistry.

Finally, we would like to underline the genuine personal character of Enzo, his ability to be a friend for his students, co-workers and colleagues, his helpful attitude and his broad cultural interests. For a long time, Enzo was a teetotaller, but over the last 20 years, he has become able to appreciate a glass of good wine. For this reason, we will accompany our wishes for his 60th birthday by opening a bottle of the best Italian wine in order to toast with all the numerous friends he has in the world. Forza Enzo, sono solo i primi sessant'anni! (Go Enzo, these are just the first 60 years!)

We are grateful to the many authors who contributed to this special issue of TCA dedicated to the 60th birthday of Enzo Barone and to the numerous referees who have dedicated their valuable time to evaluate the manuscripts. We are also grateful to Christopher Cramer for his enthusiastic acceptation of our proposal for this special issue and to the TCA staff for its help all along the editorial work. 